\title{
Investigation in optimisation of accuracy with non-contact systems by influencing variable processes
}

\author{
Ranveer S. Matharu ${ }^{1}$, Wayne Sadler ${ }^{1}$, Bekim V. Gashi ${ }^{1}$ and Trevor Toman ${ }^{1, *}$ \\ ${ }^{1}$ Institute for Future Transport and Cities, Coventry University, Gulson Road, Coventry, West \\ Midlands, CV1 2JH, United Kingdom
}

\begin{abstract}
The use of 3D scanning systems is becoming increasingly popular and an essential tool for manufacturers for inspection and measurement. With such systems being utilised on the manufacturing shop floor due to their portability and ease of use, it is no doubt that such systems are designed to address a variety of users whom, with minimal training can operate the equipment. Due to continuing demands of highquality products there is the need for manufacturers of 3D scanning systems to develop technologies that deliver fast and accurate information. However, one of the key challenges lies not in the training of people to use the equipment, but to develop engineers who can produce traceable, accurate and precise results with a declared statement of confidence quantifying the quality of the measurement. This statement of the quality of the output results relies on employing a set of workflow actions that involve planning, capture, processing and analysis, and finally output. This paper sets out to show how the results from a set of workflow actions from different categories of 3D scanning devices affects the quality of output.
\end{abstract}

\section{Introduction}

The use of 3D scanning technologies continues to play an important role as a measurement tool for comparison and verification. Advancements in terms of their portability, ease of use, decreasing costs and robustness has shown to have a global effect on their adoption into new environments. One such environment where 3D scanning technologies are making significant inroads is in manufacturing with such technologies becoming a common tool on the shop floor or assembly line. Their intuitiveness and ease of use make such 3D scanning technologies accessible to a variety of users of whom, with minimal training are able to operate the equipment thereby making it seen as an indispensable tool for many manufacturers.

While there are many benefits of 3D scanning in manufacturing, such as, the ability to quickly gather part data, the ability to shorten cycle times in product design and prototyping and the ability to provide comparatively reliable data for part accuracy and design verification, 3D scanning systems are still constrained to the limits of todays technologies.

\footnotetext{
*Corresponding author: aa2406@coventry.ac.uk
} 
As manufacturers of 3D scanners push the boundaries of sensor performance of which are governed by the laws of physics [1] it is arguable that their performance in real-working conditions is what contributes to their uncertainty. In many cases it is the manufacturers of such systems that employ their own qualification and verification routines in controlled environments to benchmark the performance of their system, and this therefore makes it challenging to relate these results to other commercially available 3D scanners. Furthermore, standards such as the VDI/VDE 2634 that manufacturers of 3D measurements systems rely on for scanner evaluation are solely based on acceptance and reverification tests carried out on three objects: a sphere, ball-bar and plane all of which are proposed to be of diffuse reflecting materials for optimal measurement [2]. Although these tests are useful for benchmarking the performance of a 3D system, they are only valid for the objects that they were tested for and with no specifications for the objects this makes comparison of results across different $3 \mathrm{D}$ scanner manufacturers challenging [3].

With the continuing demands of high-quality products, there is a need for accuracy in today's 3D scanning systems and one of the key challenges lies not in the training of people to use the equipment, but to develop engineers who can deliver information that is traceable, accurate and precise with a declared statement of confidence quantifying the quality of the measurement. This statement of the quality of the output results relies on employing a set of workflow actions that involve planning, capture, processing and analysis, and finally output. This paper aims to address the challenges and tasks associated with producing traceable measurements using portable non-contact measurement processes to produce task specific uncertainty budgets that evidence traceability. In the worlds of metrology and research tasks of defining and understanding measurement systems and measurement data has the been the domain of the metrologist. Greater degrees of confidence in measurement and therefore priory knowledge of data capture for improvements to future measurement and analysis methods.

\section{Materials and methods}

To address the challenges associated with non-contact measurement processes, two laser line scanners were used on a sandblasted machined artefact with distinctive features that included a bore and a sphere as shown in Fig. 1. The first of these scanners was a handheld MetraScan 750 from Creaform and the other a fixed scanner mounted on a 3D coordinate measurement machine (CMM), Zeiss LineScan 2-25.

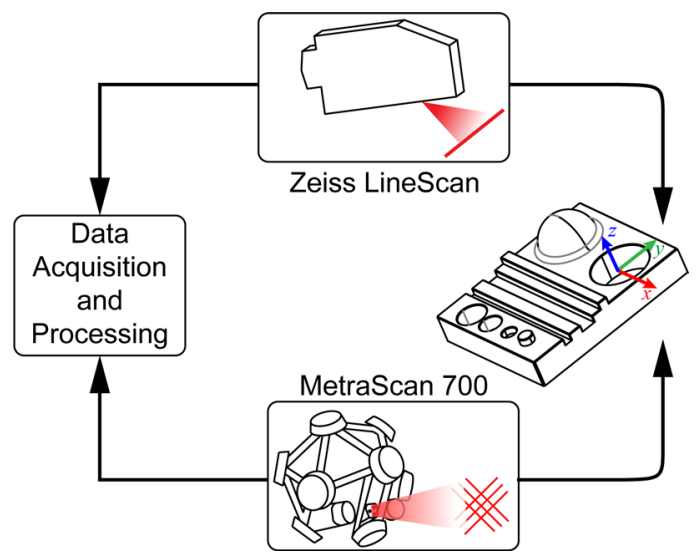

Fig. 1. Experimental setup describing the measurement process 
The first of the scanners (MetraScan 750), was a device that used 7 laser crosses located on a measuring head and was paired to an instrument that tracked its location within a measurement volume. The advantage of this type of setup, was that it allowed for a broader measurement space of $16.6 \mathrm{~m}^{3}$. With this scanner, the measurement artefact was placed on a rotating table and rotated around the part y-axis table at a rotation of approximately 2 rotations per minute. This orientation was used due to the part being held vertically, in the $y$-axis, in the rotary table chuck.

The second of the scanners (Zeiss LineScan 2-25) was a single line device fixed to an RDS articulating probe holder on a CMM (Contura G2 CMM). This setup was used to move the laser line scanner in controlled directions over the artefact. In this setup, the artefact was placed on the CMM table and a series of three scans in the YZ projection at angles $90^{\circ}, 75^{\circ}$ and $105^{\circ}$ were taken to cover the sides and front face of the artefact and similarly a series of two scans a ZX projection at angles $15^{\circ}$ and $-15^{\circ}$ to cover the top and bottom. In both instances data recording was carried out in the form point clouds using laser triangulation. The specifications of each instrument are outlined in Table 1.

Table 1. Technical data for the two acquisition systems

\begin{tabular}{|c|c|c|}
\hline $\begin{array}{c}\text { Technical } \\
\text { Specifications }\end{array}$ & $\begin{array}{c}\text { LineScan } \\
\mathbf{2 - 2 5}\end{array}$ & $\begin{array}{c}\text { MetraScan } \\
\mathbf{7 5 0}\end{array}$ \\
\hline $\begin{array}{c}\text { Resolution } \\
(\mathrm{mm})\end{array}$ & 0.02 & 0.05 \\
\hline $\begin{array}{c}\text { Measuring rate } \\
\text { (points/sec) }\end{array}$ & 700,000 & 480,000 \\
\hline $\begin{array}{c}\text { Measuring } \\
\text { range (mm) }\end{array}$ & 25 & $275 \times 250$ \\
\hline
\end{tabular}

The proposed method for understanding the performance of the instruments was carried out by measuring the artefact at different locations. Current existing standards such as the VDE 2634 [4] typically state 5 to 7 different locations. For the case of the Zeiss LineScan a measurement was taken at 5 locations and this is shown in Fig. 2(a).

Similarly, for the MetraScan 750, measurements were taken for a set of processes. The first process involved holding the instrument at 3 static locations with the artefact under rotation and gathering measurement data. In total, the artefact was rotated for three full rotations with set scans at three positions each taking place per revolution. This is shown by solid lines in Fig. 2(b) where the position that the laser scanner was handheld was in the YZ projection at the following angles $60 \pm 5^{\circ}, 0 \pm 5^{\circ}$ and $-60 \pm 5^{\circ}$.

The second process involved measurements taken with the instrument over a guided continuous arc movement. This is shown by the dashed line in Fig. 2(b) where again, the laser scanner was handheld in the YZ projection with the artefact rotated for three full rotations with each revolution representing an arc motion (down and up) of data gathered.

Finally, the third process involved measurements taken where the laser scanner was handheld and guided randomly over the artefact with focus to ensure that the part was covered thoroughly with every sweep of the laser scanner. In this instance the artefact was static. 


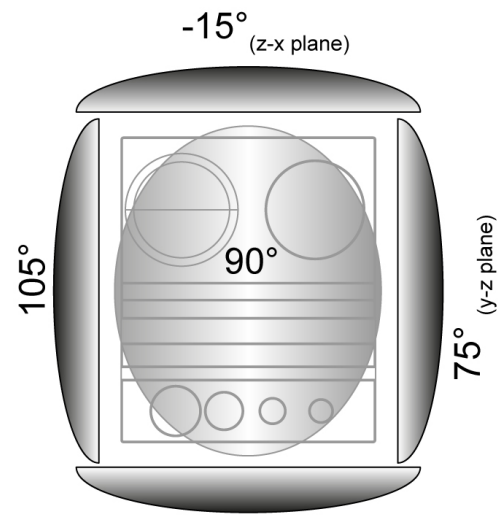

$15^{\circ}$

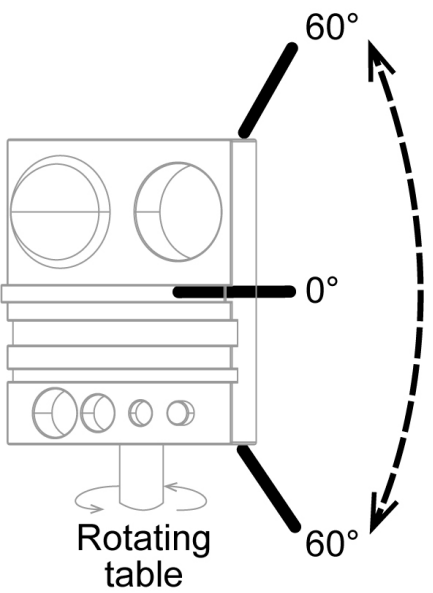

(b)

Fig. 2. Locations of measurement of the artefact carried out on the (a) Zeiss LineScan 2-25 (b) MetraScan 750.

\subsection{Data Processing}

The digitisation of the parts captured by each of the scanning systems is shown by the plots in Fig. 3 with sample cloud point data shown for the Zeiss LineScan and MetraScan 750. For the case of the Zeiss LineScan only the top face of the artefact was scanned, this was due to access constraints of the RDS articulating probe holder with the underside of the part. With the MetraScan 750 on the other hand, having the artefact mounted on a rotating table allowed for a much larger coverage area allowing for full coverage as shown by Fig. $3(\mathrm{~b})$.

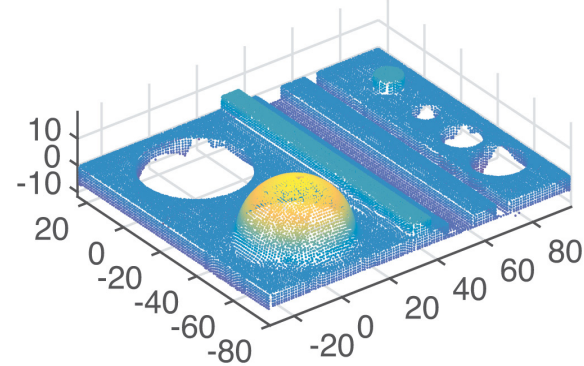

(a)

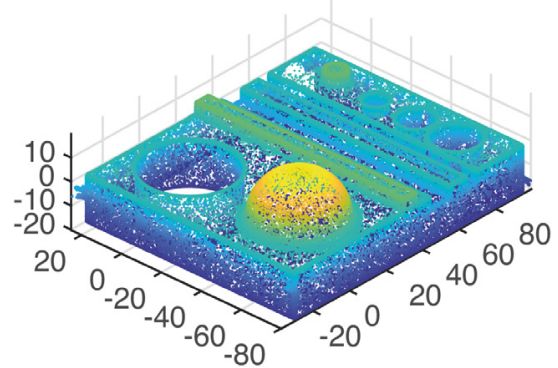

(b)

Fig. 3. Sample cloud point data obtained from (a) Zeiss LineScan (b) MetraScan 750.

The results captured from both the LineScan and the MetraScan 750 were processed in Polyworks. The imported point cloud data was first aligned to a common coordinate system with the CAD model as illustrated by the $x-y-z$ coordinate system in Fig. 1. Once aligned, extracted features of interest to be compared to each other were recorded. These features of interest were diameters of the bore and the hemisphere where a cylinder and sphere were best fitted to the point cloud data respectively. Selected regions of points for fitting a cylinder on the bore and sphere on the hemisphere were kept consistent between cloud point datasets. 


\section{Results}

The results of the measured features, namely the diameters of the bore and sphere, are shown and discussed. For the LineScan and MetraScan 750, experiments were carried as described in Section 2. For the case of the MetraScan 750, three processes were carried out. These were, a set of measurements with the scanner handheld at fixed locations, measurements over an arc motion and measurements through random focussed movements, ensuring good coverage of the bore and sphere features on the artefact.

For measurements carried out using a controlled process with the scanner handheld at fixed locations, the values obtained are relatively stable. This is particularly true for the case of the diameter of the bore and for the diameter of the sphere, with the exception of a few outliers. In the case of moving the scanner in an arc like motion, the results show poor stability in measurement across both bore and sphere diameters. This instability is due to a lack of static control in the data collection, with points being gathered "in motion" at all times. In terms of measurement stability, the process that achieved the best result was utilising the MetraScan 750 scanner, handheld over random but focussed movements across the static artefact and suggests that with prior directive, the user will consciously dwell, both in time and intensity, at specified or critical locations, gathering more than sufficient data at the relevant areas.

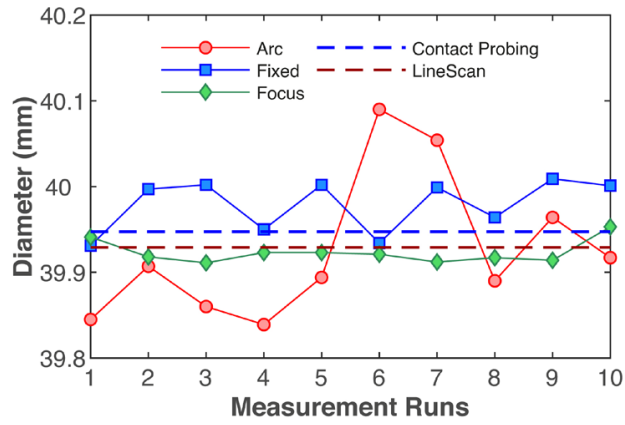

(a)

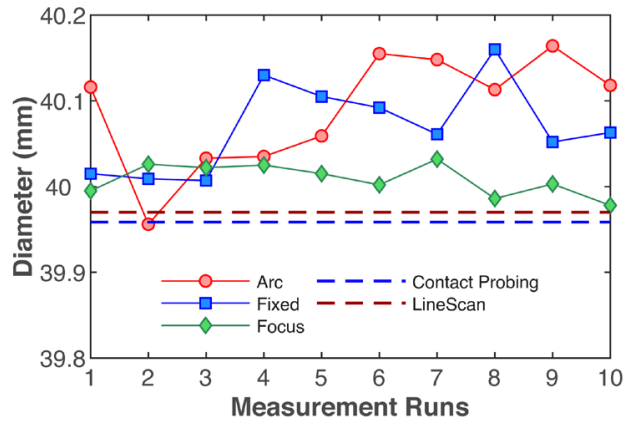

(b)

Fig. 4. Diameters of features measured for (a) Bore (b) Sphere. The lines with markers represent measurements carried out on a MetraScan 750 for a set of processes. The dashed lines represent a measurement taken using two measurement heads, a contact probe and single laser line scanner (LineScan) both of which interface with a Zeiss Contura G2 Coordinate-measuring machine.

In a monitored environment with controlled probing paths, hence repeatable data collection, the point cloud from the LineScan indicates that the bore measurement is stable, but consistently undersize. In the same conditions and, with the same stable data collection, the sphere measurement is consistently oversize. This is similarly the case with the contact probe data.

The deviations across the bore and sphere diameters are shown in Fig. 5. The graphs show deviations from nominals of $40 \mathrm{~mm}$ and show that having a process with the handheld scanner moving in an arc like fashion results in the highest spread. Focussing and having the handheld scanner fixed to capture bore and sphere show a relatively tight distribution of values that are closely resembled. 


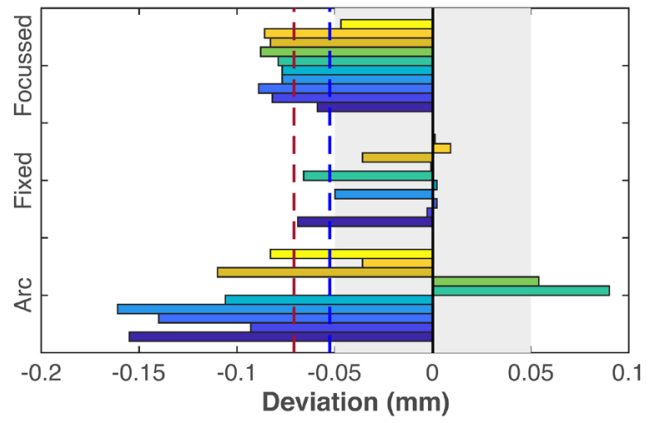

(a)

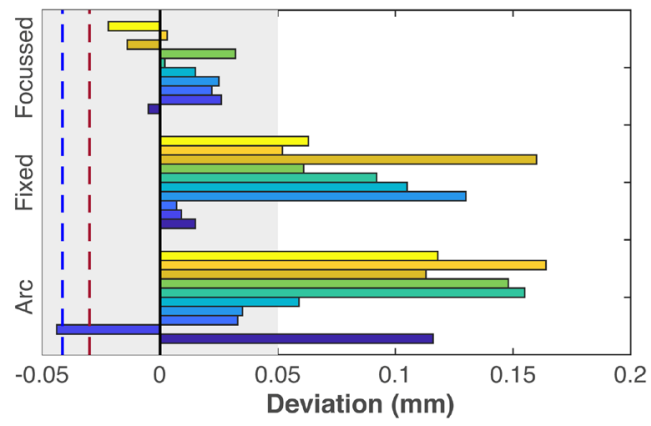

(b)

Fig. 5. Plotted deviations across (a) Bore (b) Sphere for all the measurement runs carried out on the MetraScan 750. Deviations are calculated with respect to nominal bore and sphere diameters of 40 $\mathrm{mm}$. The red dashed line represents the deviation of a LineScan measurement from a nominal of 40 $\mathrm{mm}$. The blue dashed line represents the deviation of a contact probe measurement from a nominal of $40 \mathrm{~mm}$. The shaded grey area on each graph represents a band of $\pm 0.05 \mathrm{~mm}$.

\section{Conclusions}

The results presented show that the method of a focussed measurement process using the handheld scanner, shows better results overall than employing a typical measurement strategy approach. Further investigations into limitations and trade-offs such as active scanning time periods and large data resource management need to be performed against traceable artefacts and datasets. It is clear from the results that employing a feature specific knowledge and approach would yield better results of certain features including the reference system and one might need to adopt a different approach when dealing with such features.

Further studies will involve investigating the relationship between feature sets and their their datums and the effect of varying measurement processes on them.

\section{References}

1. G. Häusler, S. Ettl, In: Leach R. (eds) Optical Measurement of Surface Topography. Springer, Berlin, Heidelberg (2011).

2. M. Carfagni, R. Furferi, L. Governi, M. Servi, F. Uccheddu and Y. Volpe, IEEE Sensors Journal, 17, pp. $4508-4519$ (2017).

3. J. R. Hodgson, P. Kinnell, L. Justham, N. Lohse, M. R. Jackson, Optics and Lasers in Engineering, 91 (2017).

4. VDI/VDE 2634 Part 2: 2002 Optical 3-D measuring systems, Optical systems based on area scanning. 